\title{
Whom to screen? A view on lung cancer screening from the pneumologist's perspective
}

\author{
Malte Asshoff $(\mathbb{D})$ Günter Weiss · Ivan Tancevski
}

Received: 23 April 2019 / Accepted: 23 May 2019 / Published online: 5 June 2019

(C) The Author(s) 2019

\begin{abstract}
Summary The benefits of screening programs are highly dependent on risk group definition. This is especially true for lung cancer screening. Results from the National Lung Cancer Screening Trial (NLST) and the NELSON trial (Dutch/Belgian randomised lung cancer screening trial) demonstrated that lung cancer screening using low-dose CT scans results in reduction of cancer-related mortality. This article gives a short overview on participant selection in the NLST and NELSON trials, and on current evidence for implementation of multivariable risk prediction models.
\end{abstract}

Keywords Lung cancer · Lung cancer screening • Low-dose CT screening $\cdot$ NLST $\cdot$ Risk prediction model

\section{Take home message}

- The National Lung Cancer Screening Trial (NLST) and NELSON trial (Dutch/Belgian randomised lung cancer screening trial) showed that lung cancer screening using low-dose CT is feasible and reduces disease associated mortality

- Smoking history is the most important factor for the definition of the high-risk group

- Lung cancer risk prediction models may show better performance than the NLST criteria

- Multivariable risk prediction models should be used for selection of patients at risk to be included in lung cancer screening programs

\footnotetext{
M. Asshoff $(\bowtie) \cdot G$. Weiss $\cdot$ I. Tancevski

Department of Internal Medicine II, Infectious Diseases,

Immunology, Pneumology, Rheumatology, Innsbruck

Medical University, Anichstraße 35, 6020 Innsbruck, Austria malte.asshoff@i-med.ac.at
}

\section{Introduction}

Worldwide, lung cancer is the leading cause of cancerrelated mortality and one of the ten most frequent causes of death [1, 2]. As with most solid malignancies, prognosis at early stages is more favourable. Unfortunately, at the time of diagnosis most patients are in an advanced stage of disease and despite recent therapeutic progress prognosis remains poor [3]. Therefore, emphasis should be set on the identification of lung cancer at an early disease stage. Due to an often asymptomatic course at early stages, screening of high-risk patients seems to be the only appropriate option to reach this goal. Thus, the aim of screening programs is the detection of clinically unapparent cancer to offer the possibility of curative treatment. So far, screening programs have been successfully established in different malignant diseases including breast, prostate, cervix or colorectal cancer. In contrast to these programs, age as the main inclusion criterion appears to be of minor relevance for lung cancer risk. Since potential benefits of disease screening are highly dependent on proper risk stratification, smoking history as most important risk factor must be included.

\section{Lung cancer screening trials}

Until recently, the National Lung Cancer Screening Trial (NLST) was the first randomized prospective trial demonstrating a significant benefit of lung cancer screening in high-risk individuals. In NLST, annual low-dose CT (LDCT) screening led to a $20 \%$ reduction in mortality from lung cancer, compared to annual screening with chest x-ray [4]. High-risk individuals were defined according to age and smoking history (age between 55-74 years and a history of smoking 
with at least 30 pack-years [PY] or, if former smokers, cessation $<15$ years).

At the 19th IASLC World Conference on Lung Cancer first survival results from a large Dutch/Belgian randomized, population-based lung cancer screening trial, the NELSON study, were presented [5]. This trial confirmed the benefits of LDCT screening in high-risk participants, compared to standard of care. According to the survival analysis at 10 years, LDCT screening led to a reduction of lung cancer-related mortality of $26 \%$ in men and of $39 \%$ in women, respectively. According to the study design, more than 15,000 participants were randomized for LDCT screening, with 4 screening rounds (year after randomization: 1, 2, 4 and 6.5) or observation.

The inclusion criteria were age between $50-75$ years, and a history of smoking with a minimum amount of 15 cigarettes per day for 25 years or 10 cigarettes for 30 years and smoking cessation of less than 10 years.

In both trials, the inclusion criteria were based on smoking history and age. In comparison to the NLST, there was a lower smoking threshold in NELSON (30 PY vs 15 PY). Most importantly, based on the NLST results, the United States Preventive Services Task Force (USPSTF) recommended lung cancer screening for current and former smokers aged 55-80 [6].

\section{Risk prediction models}

Besides smoking and age, other risk factors for lung cancer include family history, asbestos exposure and chronic lung diseases. Thus, incorporating other risk factors than smoking or age leads to a more precise definition of patients at risk for lung cancer.

In a retrospective analysis of the NLST data, participants were divided into risk quintiles using the Prostate, Lung, Colorectal and Ovarian (PLCO) model [7]. Efficacy of LDCT screening increased according to risk quintile. In quintile 1, including patients with the lowest risk, 0.2 lung-cancer deaths per 10,000 person-years were prevented, compared to 12.0 in quintile 5. Furthermore, the false-positive rate declined in higher quintiles. Screening of subjects in quintile 1 accounted for $1 \%$ of screening-prevented lung cancer deaths as compared to $88 \%$ of screening prevented death in quintiles $3-5$ which accounted for $60 \%$ of the study population. These facts emphasize the need of further adjustment of high-risk group definition. In this regard, different multivariable risk prediction models have been published, e.g. the Spitz [8], Bach [9], Knoke [10], Two-Stage Clonal Expansion (TSCE) [11], Liverpool Lung Project version $2\left(\mathrm{LLP}_{\mathrm{v} 2}\right)$ [12] and PLCO $_{\mathrm{M} 2012}$ [13] model. TenHaaf et al. did an external validation of nine risk models comparing their performance in regard to discrimination, calibration and clinical usefulness for selection of lung cancer screening candidates [14]. All models had a better specificity and sensitivity compared to the criteria used in the NLST, with appropriate risk thresholds.

Besides the implementation of additional lung cancer risk factors, a better performance of risk prediction models is mainly driven by the inclusion of a more detailed assessment of smoking history. NLST and NELSON used the risk factor in a dichotomized way ( $>$ PY threshold; <quit time duration), whereas the $\mathrm{PLCO}_{\mathrm{M} 2012}$ among others includes four smoking variables. Also, the TSCE and Knoke model, consisting solely of age and gender, but with a detailed smoking history, outperform the NLST criteria. Overall, the $\mathrm{PLCO}_{\mathrm{M} 2012}$ had the best performance in all parameters investigated, followed by Bach and TSCE.

In an external validation of the risk prediction models on data from the European Prospective Investigation into Cancer and Nutrition (EPIC) study [15], the $\mathrm{PLCO}_{\mathrm{M} 2012}$ was the one with best performance, highest sensitivity, specificity and positive predictive value. The $\mathrm{PLCO}_{\mathrm{M} 2012}$ is a modified version of the risk prediction model used in the PLCO screening trial. The multivariable risk prediction consists of age, smoking history (status, pack-years, quit time), COPD, family history of lung cancer, level of education, BMI and chest radiograph in the previous 3 years and predicts 6 -year lung cancer risk. It was validated in the PLCO intervention group of smokers and in NLST participants [13]. Risk stratification with PLCO $_{\mathrm{M} 2012}$ improved sensitivity and positive predictive value, with equal specificity compared to NLST criteria.

Furthermore, it showed good predictive performance in an Australian population-based cohort study including 95,882 participants, being the largest independent validation of the PLCO $_{\mathrm{M} 2012}$ [16]. It was further shown that the main influence of risk prediction in PLCO $_{\mathrm{M} 2012}$ is smoking history and age, since after exclusion of other variables, PLCO $_{\mathrm{M} 2012}$ still had a better performance than the NLST criteria.

The Bach model is based on the Carotene and Retinol Efficacy trial (CARET) and includes age, gender, smoking (intensity, duration, years since cessation) and asbestos exposure [9]. In the TSCE incidence model, based on the Nurses' Health Study (NHS) and Health Professional Follow-up Study (HPFS), age, gender and a detailed smoking assessment (status, duration, intensity, time since cessation) predicted lung cancer incidence [11].

The $\mathrm{LLP}_{\mathrm{v} 2}$ estimates the lung cancer probability within a 5-year period, by using additional risk factors on top of age and smoking history, including family history of lung cancer, prior diagnosis of a malignancy other than lung cancer, prior diagnosis of pneumonia and exposure to asbestos. It was developed by analyzing data of 579 lung cancer patients and of 1157 ageand sex-matched controls [12]. Validation was done using three independent external data sets showing good discrimination [17]. Furthermore, it has been used for risk stratification and patient selection in the prospective, randomized controlled UK Lung Cancer 
Screening Trial using an $\mathrm{LLP}_{\mathrm{v} 2}$ risk threshold of $\geq 5 \%$ five-year lung cancer risk [18].

All presented models were superior to the NLST criteria in risk prediction for lung cancer. A critical issue with risk models appeared to be the definition of predictive risk thresholds for initiation of cancer screening. In the studies here presented the risk models had their individual thresholds. Fi. in the PLCO $_{\mathrm{M} 2012}$, lung cancer screening should be considered when 6 -year cancer risk was $\geq 1.51 \%$. So far, there are no recommendations which risk prediction model and risk threshold should be used for implementation in lung cancer screening programs, but available data demonstrate the superiority of risk prediction models compared to the NLST criteria.

Prospective studies are needed using risk prediction models as inclusion criteria in LDCT screening programs to evaluate if this results in an even greater benefit compared to NLST and NELSON. Importantly, chronic pulmonary diseases represent criteria which should be included. Among others, COPD and pulmonary emphysema are strong predictors of lung cancer, and represent lung cancer risk factors independent of smoking history [19]. Furthermore, lung fibrosis is associated with a substantial increased lung cancer risk, as is long-term asbestos exposure.

\section{Conclusion}

Although recent years have seen major advances in the treatment of lung cancer, prognosis is poor. Furthermore, the number of lung cancer cases is still rising, and apart from preventive measures and initiative to reduce risk factors such as smoking, new strategies for early detection of cancer are needed to reduce disease-related morbidity and mortality. The NLST and NELSON trial have proved that large scale LDCT lung cancer screening is feasible, resulting in a reduction of lung cancer-associated mortality. However, age and smoking history were the sole inclusion criteria. On the other hand, different multivariable lung cancer risk prediction models have been established. Used in retrospective analyses, these models showed a significantly better performance than the criteria used in the NLST trial. Thus, we conclude that a more sophisticated and individualized risk-based approach may be more efficient than a risk stratification based on age and smoking history only. To increase the benefit of LDCT screening, risk prediction models such as the $\mathrm{PLCO}_{\mathrm{M} 2012}$ may be implemented for identification of patients at risk. However, further studies are needed to corroborate improved performance of risk prediction models. In summary, current evidence encourages the use of risk prediction models in lung cancer screening programs.

Funding Open access funding provided by University of Innsbruck and Medical University of Innsbruck.
Conflict of interest M. Asshoff, G. Weiss, and I. Tancevski declare that they have no competing interests.

Open Access This article is distributed under the terms of the Creative Commons Attribution 4.0 International License (http://creativecommons.org/licenses/by/4.0/), which permits unrestricted use, distribution, and reproduction in any medium, provided you give appropriate credit to the original author(s) and the source, provide a link to the Creative Commons license, and indicate if changes were made.

\section{References}

1. Stewart BW, Wild CP, editors. WHO world cancer report world cancer report. 2014.

2. WHO. Global health estimates. 2016. https://www.who. $\mathrm{int} /$ news-room/fact-sheets/detail/the-top-10-causesof-death.

3. Walters S, Maringe C, Coleman MP, et al. Lung cancer survival and stage at diagnosis in Australia, Canada, Denmark, Norway, Sweden and the UK: a population-based study, 2004-2007. Thorax. 2013;68:551-64.

4. National Lung Screening Trial Research Team, Aberle DR, Adams AM, et al. Reduced lung-cancer mortality with lowdose computed tomographic screening. N Engl J Med. 2011;365:395-409.

5. DeKoning H, Van Der Aalst C, TenHaaf K, et al. Effects of volume CT lung cancer screening: mortality results of the NELSON randomized-controlled population based trial. World Conference on Lung Cancer; 25.9.2018. 2018. AbstractPL02.05.

6. VirginiaA. Moyer, MD, MPH; on behalf of the U.S. Preventive services task force. Screening for lung cancer: U.S. Preventive services task force recommendation statement. Ann Intern Med. 2014;160(5):330-8.

7. Kovalchik S, Tammemagi M, Berg C, et al. Targeting of low-dose CT screening according to the risk of lung-cancer death. NEngl J Med. 2013;369:245-54.

8. Spitz MR, Hong WK, Amos CI, et al. A risk model for prediction oflung cancer. J Natl Cancer Inst. 2007;99:715-26.

9. Bach PB, Kattan MW, Thornquist MD, et al. Variations in lung cancer risk among smokers. J Natl Cancer Inst. 2003;95(6):470-8.

10. Knoke JD, Burns DM, Thun MJ. The change in excess risk of lung cancer attributable to smoking following smoking cessation: an examination of different analytic approaches using CPS-I data. Cancer Causes Control. 2007;19(2):207-19.

11. Meza R, Hazelton WD, Colditz GA, Moolgavkar SH. Analysis oflung cancer incidencein the nurses' health and the health professionals' follow-up studies using a multistage carcinogenesis model. Cancer Causes Control. 2008;19(3):317-28.

12. Cassidy A, Myles JP, van Tongeren M, et al. The LLP risk model: an individual risk prediction model for lung cancer. BrJCancer. 2008;98:270-6.

13. Tammemägi MC, Katki HA, Hocking WG, et al. Selection criteria for lung-cancer screening. $\mathrm{N}$ Engl J Med. 2013;368:728-36.

14. TenHaaf K, Jeon J, Tammemägi MC, et al. Risk prediction models for selection of lung cancer screening candidates: a retrospective validation study. Plos Med. 2017;4(14):e1002277.

15. Li K, Hüsing A, Sookthai D, et al. Selecting high-risk individuals for lung cancer screening: a prospective evaluation of existing risk models and eligibility criteria in the German EPIC cohort. Cancer Prev Res (Phila). 2015;8:777-85.

16. Weber M, Yap S, Goldsbury D, Manners D, Tammemagi M, et al. Identifying high risk individuals for targeted lung can- 
cer screening: Independent validation of the PLCOm2012 risk prediction tool. Int J Cancer. 2017;141(2):242-53.

17. Raji OY, Duffy SW, Agbaje OF, et al. Predictive accuracy of the Liverpool Lung Project riskmodel for stratifying patients for computed tomography screening for lung cancer: a casecontrol and cohort validation study. Ann Intern Med. 2012;157:242-50.

18. Field JK, Duffy SW, Baldwin DR, et al. The UK Lung Cancer Screening Trial: a pilot randomised controlled trial of low-dose computed tomography screening for the early detection of lung cancer. Health Technol Assess. 2016;20:1-146.

19. AamliGagnat A, Gjerdevik M, Gallefoss F, Coxson HO, Gulsvik A, Bakke P. Incidence of non-pulmonary cancer and lung cancer by amount of emphysema and airway wall thickness: a community-based cohort. ERJ Open Res. 2017;49:1601162.

Publisher's Note Springer Nature remains neutral with regard to jurisdictional claims in published maps and institutional affiliations.

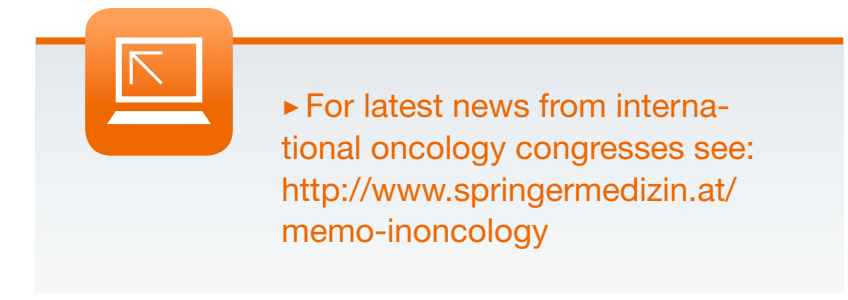

